\title{
论㓞性材料的可膨胀塑性本构方程及 分叉时塑性加载路径
}

\author{
李 国 堔 \\ (中国科学院力学研究所, 北京 100080)
}

\section{摘要}

文中证明所导出的可膨胀塑性本构方程是具有一般性的一种表达式，在此基础 上论证了固体变形出现分又时材料的本构行为总是顺从尽可能“软”的加载路径.

关键词：韧性材料,可脙胀塑性, 本构方程, 分叉, 应变钦化

\section{一、引 言}

韧性断裂是研究材料失效问题的一个重要领域。随着载荷的增大, 材料内部会出现微空 洞和剪切带，它们的扩展和聚合形成韧性损伤以致断裂 (有关这方面的实验现象可参见 $\mathrm{Ha}-$ ncock 和 Cowling ${ }^{[1]}$, Anand 和 Spitzig ${ }^{[2]}$ 的报道). 这就提出了两个至关重要的研究课题: 一是如何在连续介质本构方程中计人微空洞所造成的损伤作用和体膨胀; 二是出现剪切带状 分叉时材料行为所应遵循的加载路径.

Gurson $^{[3]}$ 在分析空洞模型的基础上修改了经典塑性力学中的 von-Mises 型势函数, 给 出含有空洞体积百分数 $f_{v}$ 的塑性加载面. 这一曲面在应力空间中随着 $f_{v}$ 值的增大而缩小, 即 出现代表内部损伤的应变软化现象.在假设正交法则仍然成立的前提下，Gurson 提出了可膨 胀塑性本构方程. 为分析剪切带型分叉, Hill 和 Hutchinson ${ }^{|4|}$ 曾提出过有关的控制方程. 但他们沿用了分析结构屈曲 (分叉) 的“一致加载” 条件, 即认为分叉时塑性加载路径不变 (参 阅文献 [5]).

但是, 近年的发展表明:（i）实际材料中除了存在有较大的空洞 (尺度为 $10 \mu \mathrm{m}$ ), 在更高 的应力/应变状态下还会出现次级空洞 (尺度为 $1 \mu \mathrm{m}$ ). 两级空洞之间有较强的交互作用并导 致材料的严重损伤. 此时, 仅含空洞体积百分数 $f$ 。的塑性加载面不再是光滑和凸的且伴随有 总体弹性模量值的明显下降 ${ }^{[6,7]}$. (ii) Anand 和 Spitzig 指明 ${ }^{2]}$, 由 Hill 和 Hutchinson 理 论 ${ }^{[4]}$ 所导出的出现剪切带的临界应变值远高于实验值.

基于上述现状, 本文提供了可膨胀塑性本构方程的理论框架, 它在形式上具有一般性的特 点;论证了含有微结构损伤的材料出现分叉现象时,将选择“软”加载路经.

本文 1989 年 11 月 27 日收到, 1990 年 6 月 6 日收到修改稿.

"文中有关工作为中国科学院力学研究所“非线性连续介质力学开放研究实验室”资助. 


\section{二、可膨胀塑性本构方程的准导}

基于对韧性损伤的微结构力学的研究, 我们曾指出 ${ }^{[6,7]}$, 由于弹性与塑性的交互作用并导 致弹性模量的急剧降低,加上塑性加载面变得不规则,传统的凸性与正交法则无从成立. 在此 情况下,欲建立合理的塑性本构方程就需借助于更一般的原则, 而不应限定由循环应力或应变 所耗散能必须保持为正的. 因此，以下的准导将建立在耗散塑性能表达式的等价性这一出发 点.

先将应力张量 $\sigma_{i}$ 和塑性应变增量 $d \varepsilon_{i j}^{(p)}$ 分别分解为各自约编量和体量部分. 在直角坐 标下将有:

$$
\begin{aligned}
& \sigma_{i j}=S_{i i}+\delta_{i j} \sigma_{m}, \\
& d \varepsilon_{i i}^{(p)}=d e_{i i}^{(p)}+\dot{o}_{i j} d \varepsilon_{m}^{(p)},
\end{aligned}
$$

其中 $S_{i j}\left(=\sigma_{i j}-\frac{1}{3} \delta_{i j} \sigma_{k k}\right)$ 和 $\sigma_{m}\left(=\frac{1}{3} \sigma_{k k}\right)$ 分别为应力偏量和平均应力, 而 $d e_{i}^{(p)}\left(=d \varepsilon_{i \prime}^{(p)}-\right.$ $\left.\frac{1}{3} \delta_{i j} d \varepsilon_{k}^{(p)}\right)$ 和 $d \varepsilon_{m}^{(p)}\left(=\frac{1}{3} d \varepsilon_{k k}^{(p)}\right)$ 则是塑性应变增量的偏量和平均部分.

耗散塑性能的增量可以表示为

$$
\begin{aligned}
d W^{(p)} & =\sigma_{i j} d \varepsilon_{i j}^{(p)}=\left(S_{i j}+\phi_{i} \sigma_{m}\right)\left(d e_{i j}^{(p)}+s_{i} d \varepsilon_{m}^{(p)}\right) \\
& =S_{i} d e_{i j}^{p}+3 \sigma_{m} d \varepsilon_{m}^{p)} .
\end{aligned}
$$

需要说明的是,这里的应力 $\sigma_{i j}$ 和塑性应变 $\varepsilon_{i j}^{(p)}$ 都是一般意义的量, 在小应变和大应变情况下 都有代表意义. 要求的条件是应力及其共轭的塑性应变增量的标量积, 可以代表耗散塑性能 的增量. 例如, 相对不同广义时间 $t$ 可以有不同构架下的 $d W^{(p)}$ 表达式. 2 可以选在 0 (衫 始), $t$ (瞬时)和 $t_{0}$ (某固定时刻). 相应地, 可以将 $d W^{(p)}$ 表示为

(a)

$$
d W_{(a)}^{(p)}=S_{i,}^{(k)} d \varepsilon_{i}^{(p)} \text {, }
$$

在 Lagrangian 坐标含义下, $S_{i j}^{(k)}$ 代表第二 Piola-Kirchhoff 应力, 又此时的 $\varepsilon_{i j}^{(p)}$ 应是塑性 的 Green 应变.

(b)

$$
d W_{t}^{(p)}=\sigma_{i j} D_{i j}^{(p)},
$$

则是 Eulerian 意义的. 此时 $\sigma_{i j}$ 专门用于 Cauchy 真应力且 $D_{i j}^{(p)}\left(=\frac{1}{2}\left(V_{i, i}+V_{i, i}\right)^{(p)}\right)$ 是变形率的塑性部分, 其中 $V_{i}$ 是速率分量.

$$
d W_{i}^{(p)}=\tau_{i j} D_{i j}^{(p)},
$$

当 up-dated Lagrangian（逐级更新拉氏体系）的描述方法被选作参考系统. 这里, $\boldsymbol{\tau}_{\boldsymbol{i}} ;$ 为 Kirchhoff 应力, 它与真应力的关系是 $\tau_{i j}=\frac{d V}{d V_{0}} \sigma_{i j}$, 其中 $d V$ 和 $d V_{0}$ 分别代表在 $t$ 和 $t_{0}$ 时 刻的体积微元.

另一方面,如果定义

$$
d W^{(p)}=\sigma_{e} d \varepsilon^{(p)}+3 \sigma_{m} d \varepsilon_{m}^{(p)},
$$

这里, 对 $\sigma_{e}$ 取名为等效应力并被定义为

$$
\sigma_{c}=\left(\frac{3}{2} S_{i j} S_{i j}\right)^{1 / 2}
$$


又称 $\varepsilon_{e}^{(p)}$ 为塑性等效应变.

比较方程式(3)和(4)可见

$$
\sigma_{e} d \varepsilon_{i}^{(p)}=S_{i j} d e_{i j}^{(p)} \text {. }
$$

既然在任意应力状态下等式(6)都应成立, 又联系(5)式中对等效应力的定义, 可以导出

$$
d e_{i j}^{(p)}=\frac{3}{2} \frac{d \varepsilon_{e}^{(p)}}{\sigma_{e}} S_{i} \text {. }
$$

于是

$$
d e_{i j}^{(p)} d e_{i}^{(p)}=\frac{9}{4} \frac{d \varepsilon_{e}^{(p) 2}}{\sigma_{e}^{2}} S_{i j} S_{i j}
$$

又

$$
d \varepsilon_{i}^{(p)}=\left(\frac{2}{3} d e_{i j}^{(p)} d e_{i}^{(p)}\right)^{1 / 2},
$$

所以,相应于等效应力的定义式(5)和任意耗散塑性能增量中偏量部分的等价表示原则, 塑性 等效应变的增量就应该取(9)式形式。

将(7)式代入(2)式后，得到

$$
\begin{aligned}
d \varepsilon_{i,}^{(p)} & =\frac{3}{2} \frac{d \varepsilon_{e}^{(p)}}{\sigma_{e}} S_{i j}+\delta_{i j} d \varepsilon_{m}^{(p)} \\
& =\frac{3}{2} \frac{d \sigma_{e}}{E_{t e}^{(p)} \sigma_{e}} S_{i j}+\delta_{i j} \frac{d \sigma_{k k}}{3 E_{i m}^{(p)}},
\end{aligned}
$$

其中 $E_{i e}^{(p)}=d \sigma_{e} / d \varepsilon_{\varepsilon}^{(p)}, E_{i m}^{(p)}=d \sigma_{m} / d \varepsilon_{m}^{(p)}$ 分别是 $\sigma_{e}-\varepsilon_{e}^{(p)}$ 和 $\sigma_{m}-\varepsilon_{m}^{(p)}$ 曲线上的塑性切线 模量. 又因

$$
d \sigma_{s}=\frac{3}{2 \sigma_{s}} S_{i i} d \sigma_{i i}
$$

方程 (10) 可改写为

$$
d \varepsilon_{i j}^{(p)}=\frac{9}{4 E_{t e}^{(p)}} \frac{S_{i} S_{k l} d \sigma_{k l}}{\sigma_{e}^{2}}+\frac{1}{3 E_{i m}^{(p)}} \delta_{i j} d \sigma_{k k}
$$

设全部应变增量为弹性部分与塑性部分之和且弹性部分遵守 Hooke 定律, 于是

$$
\begin{aligned}
d \varepsilon_{i j} & =d \varepsilon_{i j}^{p)}+d \varepsilon_{i j}^{p^{\prime}} \\
=\frac{1}{E}\left[(1+\nu) d \sigma_{i 1}-\nu \delta_{i j} d \sigma_{k k}\right] & +\frac{9}{4 E_{t e}^{(p)}} \frac{S_{i j} S_{k l}}{\sigma_{e}^{2}} d \sigma_{k l} \\
& +\frac{1}{3 E_{l m}^{(p)}} \delta_{i j} d \sigma_{k k},
\end{aligned}
$$

其逆形式为

$$
\begin{aligned}
d \sigma_{i t}= & \frac{E}{1+\nu}\left[\frac{1}{2}\left(\delta_{i k} \delta_{i l}+\delta_{i l} \delta_{i k}\right)+\delta_{i j} \delta_{k l} \frac{\nu-E I}{1-2 \nu} \cdot \frac{E_{l m}^{(p)}}{E / E_{l m}^{(p)}}\right. \\
& \left.-2 \sigma_{e}^{2} \quad E_{l p}^{(p)} \frac{S_{i j} \delta_{k l}}{\frac{2}{3}(1+\nu)+E / E_{i e}^{(p)}}\right] d \varepsilon_{k l \cdot}
\end{aligned}
$$

方程 (13),(14)与 Li-Howard ${ }^{\text {(8) }}$ 所提出的可膨胀塑性本构方程具有相同的形式, 其中 $E$ 和 $\nu$ 分别是杨氏模量和 Poisson 系数. 以上推导的过程表明,方程 (13)和(14)不涉及塑性势函数/ 
正交法则。它们仅是基于耗散塑性能增量在表达形式上的等价变换。显然，这种表达形式的 变换可以不是唯一的, 但仍然具有一般性的性质. 因为它既不受传统塑性力学中所包含的约 束的限定, 也不需要满足䛇环应力/应变所耗散的能量必须为正的条件 ${ }^{[0]}$. $E_{l m}^{(p)}$.

根据材料中有无损伤, 我们将区分两类主要情况来讨论如何确定塑性切线 模 量 $E_{i c}^{(p)}$ 和

\section{1. 无损伤的塑性应变}

一般来说这是指韧性材料处于小应变情况. 此时, $E_{i m}^{(p)} \rightarrow \infty$ 且方程 (13), (14) 退化为 Prandtl-Reuss 关系式. 在这种情况下，Drucker 公设及其相应的凸性和正交法则是有效 的. 进而可以假设加载面与 von-Mises 届服面具有相似的形状. 每一个加载面含有固定的 等效应力值并与塑性等效应变之间存在着一一对应的关系. 按照这一规程可以认为塑性切线 模量 $E_{i e}^{(p)}$ 是 $\varepsilon_{c}^{(p)}$ 的单值函数. 于是, 利用单轴加载下的应力-应变曲线 $\sigma-\varepsilon$ 就可以确定这 种关系. 这是因为

$$
\frac{1}{E_{t e}^{(p)}}=\frac{1}{E_{t}}-\frac{1}{E}
$$

其中, $E_{t}=d \sigma / d \varepsilon, \sigma_{e}=\sigma$ (单轴应力)又 $\varepsilon_{c}^{(p)}=\varepsilon^{(p)}$ (单轴上应变中的塑性部分).

为区分塑性加载与弹性卸载, 传统塑性力学已针对这一情况作了明文规定, 这里不再赘 述.

\section{2. 含有损伤的塑性应变}

当应变逐渐增大, 㓞性损伤将在塑性应变中起作用. 在这种情况下, 可以设想 $E_{t c}^{(p)}$ 和 $E_{m}^{(p)}$ 是两个标量函数. 它们取决于应力状态 $\boldsymbol{\sigma}$, 塑性应变状态 $\boldsymbol{\varepsilon}(p)$ 和表征损伤作用的内变 量参数组成的向量 $\boldsymbol{K}_{1}, \boldsymbol{K}_{2}$. 这就是说

$$
E_{i c}^{(p)}=F_{1}\left(\boldsymbol{\sigma}, \boldsymbol{\varepsilon}^{(p)}, \boldsymbol{K}_{1}\right), E_{i m}^{(p)}=F_{2}\left(\boldsymbol{\sigma}, \boldsymbol{\varepsilon}^{(p)}, \boldsymbol{K}_{2}\right) .
$$

基于针对空洞型损伤所作的微结构模型分析和实验研究 ${ }^{[1,6,10-12]}$, 可以知道应变软化效应 和塑性可膨胀性是损伤行为的两个主要特征. 由于剪切型损伤并不一定附有明显的体积变化, 所以最好对偏量和体量两部分响应作分别处理. 关键的一环就是如何在偏量和体量的各自空 间中确定由应变硬化转为应变软化的转折点. 就体量空间而言, 在出现应变软化之前的膨胀 量是可以忽略的. 在此, 再一次利用从空洞损伤研究中所获取的知识和准则, 可以建议

(a) 在 $\sigma_{e}-\varepsilon_{t}$ 曲线上,当

时则出现应变软化, $E_{t e}$ 由正转为负值.

$$
\sigma_{m}+\lambda_{\varepsilon} \sigma_{e}=\sigma_{c e} \text { 或 } \varepsilon_{s}=\varepsilon_{\varepsilon s}
$$

(b) 在 $\sigma_{m}-\varepsilon_{m}$ 曲线上,当

时, 则 $E_{l m}$ 成为负的有限值.

$$
\sigma_{m}+\lambda_{m} \sigma_{m}=\sigma_{c m}
$$

(c) 当

$$
\varepsilon_{n}+i \varepsilon=\varepsilon_{c},
$$

则表明材料失效. 经承受了相当程度的应变软化之后, 材料已严重损伤并丧失了支承作用.

在 $(17),(18)$ 和 (19) 式之中; $\lambda_{e}, \sigma_{c e}, \varepsilon_{c e}, \lambda_{m s}, \sigma_{c m}, \lambda, \varepsilon_{c}$ 均为待定的材料常数联同应变软化 以后的 $E_{c c}$ 和 $E_{t m}$, 各值一起构成描述材料中韧性损伤和失效所需要的参数也是 (16) 式的 
具体形式. $\lambda_{e}, \lambda_{m}$ 和 $\lambda 3$ 个参数是起着平衡偏量部分和体量部分的应力或应变的作用. 又由 于在大应变情况下全部应变值与塑性部分的差别可以忽略, 这里和以后我们取消了代表塑性 部分的标记 $(p)$.

为确定以上各参数, 在文献 [12]中作了报道和示范,所采用的方法是将计算机模拟与宏观 的和微结构的实验数据相结合. 如前面所概述, 微结构研究的确可以帮助我们认识损伤机理, 从而建立描述韧性材料损伤后的本构框架及选择最必须的有关参数. 然而, 内部机制是如此 地复杂 ${ }^{[6]}$, 不能设想不依靠实验数据就能提供定量的材料损伤的评估. 这就是为什么我们要 强调将计算模拟与实验信息相结合的方法 ${ }^{[12]}$.

\section{三、分叉时的塑性加载路径}

众所周知,分叉条件可以归结为泛函 $\pi$ 的二次变分 $Q$ 为零,即 $Q$ 达到驻值,于是

$$
\delta^{2} \pi=Q=0 .
$$

由于选用的参考体系不同,(20)式的具体形式可以不同,但本质上可以将增量型表达式在物体 体积 $v$ 内归结为

$$
Q=\int_{0}\left[\delta\left(d \sigma_{i j}\right) \delta\left(d \varepsilon_{i j}\right)+F(\sigma, \delta V)\right] d \nu,
$$

其中应力和应变的含义, 和(3)式一样, 取决于所选用的坐标系统. 对于 Lagrangian 和 updated Lagrangian 体系,可参见文献 [13]和[14], 其中都有 $Q$ 的具体形式.（21)式中的前一项 代表应力增量与应变增量的一次变分之间的标量积. 后一项 $F$ 是应力状态 $\boldsymbol{\sigma}$ 和速率变分 (或 称扰动) $\delta \boldsymbol{V}$ 的标量函数，它的具体形式取决于参考体系但并不影响以下的证明过程.

设物体承受着任意几何可能的扰动 $\delta \boldsymbol{V}$. 出现分又时, 可以讨论以下几种加载路径:

(a) 直到分叉前所形成的塑性区,在分叉时部分地改变为弹性卸载区;

（b）原塑性区保持为塑性的;

（c）原塑性区中出现应变软化;

（d）原塑性区中不仅出现应变软化而且还萌发塑性体膨胀.

在小应变情况下,由于没有损伤,讨论 (a) 和 (b) 两种加载路径已满足要求。但在塑性应 变增大并出现损伤以后, 则 (c) 和 (d) 也成为可能,这是至今尚未讨论的. 例如, 实验现象表 明 ${ }^{[1]}$, 联接大空洞的剪切带是由细小空洞所串连. 从折合的连续体来看, 这个带内材料不仅会 出现应变软化而且还有塑性体膨胀。

利用 (14)式, 可以将(21)式中应力增量变分在分叉时表示为

$$
\begin{aligned}
\delta\left(d \sigma_{i j}\right)=L_{i j k l}^{*} \delta\left(d \varepsilon_{k l}\right) & \\
L_{i, k l}^{*}= & \frac{E}{1+v}\left[\frac{1}{2}\left(\delta_{i k} \delta_{i l}+\delta_{i l} \delta_{i k}\right)+\delta_{i j} \delta_{k l} \frac{\nu-E / 3 E_{l m}^{*}}{1-2 \nu+E / E_{l m}^{*}}\right. \\
& \left.-\frac{3}{2 \sigma_{e}{ }^{2}} \frac{E}{E_{t e}^{*}} \frac{S_{i j} S_{k l}}{\frac{2}{3}(1+v)+\frac{E}{E_{l e}^{*}}}\right] .
\end{aligned}
$$

为区分分叉时的切线模量与分叉前的有关量, 在此均附加一个“*”号.

对应以上 4 种可能的加载路径, 将 $(22 \mathrm{a}, \mathrm{b})$ 式代人(21)式后, 可以得到 $Q_{a}, Q_{b}, Q_{c}$ 和 $Q_{d}$. 可以证明 
先设整个物体体积

$$
Q_{a} \geqslant Q_{b} \geqslant Q_{c} \geqslant Q_{d}
$$

$$
v=v_{s}+v_{c},
$$

其中 $v_{s}$ 是各种加载路径相互比较时所共有的弹塑性区, 而 $v_{c}$ 则是出现不同加载路径的区域. 于是可以写

$$
\begin{array}{ll}
Q_{a}=Q_{s}+\Delta Q_{a}, & Q_{b}=Q_{s}+\Delta Q_{b}, \\
Q_{c}=Q_{s}+\Delta Q_{c}, & Q_{d}=Q_{s}+\Delta Q_{d},
\end{array}
$$

这鉦

$$
Q_{s}=\int_{\nu,} L_{i k_{k l} \delta} \delta\left(d \varepsilon_{i j}\right)\left(d \varepsilon_{k l}\right) d v_{s}+\int_{。} F(\sigma, \delta \boldsymbol{V}) d v .
$$

在不同加载路径相互比较时, $v s$ 区是共同的且没有软化或膨胀突变现象, 因此刚度系数张量 $L_{i j k l}$ 上无需附加 “ $*$ ”号. 又由于是讨论同一个（但是任意可能的）抗动 $\delta \boldsymbol{V}$ 和相同的分叉前 应力 $\boldsymbol{\sigma}$ 状态, 于是在整个体积 $V$ 内 $F$ 函数的积分无论采用何种参考体系均不影响 $(23)$ 式的比 较. $v_{s}$ 域内的积分也是共同的,不参于 (23)式的比较.

只要将注意力集中在比较 $\Delta Q_{a}, \Delta Q_{b}, \Delta Q_{c}$ 和 $\Delta Q_{a}$, 它们的具体形式是

$$
\begin{aligned}
& \Delta Q_{a}=\int_{0} L_{i, k l}^{*(d)} \delta\left(d \varepsilon_{i j}\right) \delta\left(d \varepsilon_{k l}\right) d v_{c}, \\
& \Delta Q_{b}=\int_{0} L_{i ; k l}^{*(b)} \delta\left(d \varepsilon_{i j}\right) \delta\left(d \varepsilon_{k l}\right) d v_{c}, \\
& \Delta Q_{c}=\int_{0} L_{i, k l}^{*(c)} \delta\left(d \varepsilon_{i i}\right) \delta\left(d \varepsilon_{k l}\right) d v_{c}, \\
& \Delta Q_{d}=\int_{0} L_{i, k i}^{* i d} \delta\left(d \varepsilon_{i j}\right) \delta\left(d \varepsilon_{k l}\right) d v_{c} .
\end{aligned}
$$

根据 4 种可能的加载路径,由(22b)式可知

(a)

$$
L_{i ; k l}^{*(a)}=\frac{E}{1+\nu}\left[\frac{1}{2}\left(\delta_{i k} \delta_{i l}+\delta_{i l} \delta_{i k}\right)+\frac{\nu}{1-2 \nu} \delta_{i} \delta_{k l}\right],
$$

(b)

$$
L_{i, k l}^{*(b)}=L_{i, k l}^{*(a)}-\frac{3}{2 \sigma_{:}^{2}}, \frac{s_{i /} S_{k l}}{1+\frac{2}{3}(1+\nu) E_{t e} / E}, E>E_{t:} \geqslant 0,
$$

(c)

$$
L_{i j k^{\prime}}^{*\left(c^{\prime}\right)}=L_{i, k l}^{*(a)}-\frac{3}{2 \sigma^{i}} \frac{S_{i j} S_{k l}}{1+\frac{2}{3}(1+\nu) E_{*}^{*} / E},-E<E_{l e}^{*} \leqslant 0,
$$

(d) $\quad L_{i, k l}^{*(d)}=L_{i k l}^{*}$ (见 (22b) 式) 且 $E_{m}^{*}$ 为有限值.

将 (28a) 和 (28b) 式分别代入 $(27 d)$ 和 (27b) 式, 经比较可以看出

$$
S_{i} S_{k l} ;\left(d \varepsilon_{i i}\right) \delta\left(d \varepsilon_{k l}\right)=\left[S_{i} ; \delta\left(d \varepsilon_{i j}\right)\right]^{2}>0 .
$$

又因 $1+\frac{2}{3}(1+\nu) E_{t e} / E>0$,

于迹 $L_{i ; k l}^{* i b} \delta\left(d \varepsilon_{i j}\right) \delta\left(d \varepsilon_{k l}\right)=L_{i, k l}^{*(\boldsymbol{z})} \delta\left(d \varepsilon_{i j}\right) \delta\left(d \varepsilon_{k l}\right)-$ (正值)

$$
\leqslant L_{i j k}^{*(a)} \delta\left(d \varepsilon_{i j}\right) \delta\left(d \varepsilon_{k l}\right) \text {. }
$$

只有当 $E_{t e} \rightarrow \infty$, 即没有塑性应变情况下, (29)式的左右两端才会相等. 由此, 在 $v_{c}$ 体积内 
积分后, 应得到结论

$$
\Delta Q_{0} \geqslant \Delta Q_{b} .
$$

依此类推,比较 $(28 b)$ 和 (28c) 式可见,由于

$$
\left[1+\frac{2}{3}(1+\nu) E_{t e} / E\right] \geqslant\left[1+\frac{2}{3}(1+\nu) E_{t e}^{*} / E\right],
$$

那么,

$$
L_{i j k l}^{*(c)} \delta\left(d \varepsilon_{i j}\right) \delta\left(d \varepsilon_{k l}\right) \leqslant L_{i j k l}^{*(b)} \delta\left(d \varepsilon_{i j}\right) \delta\left(d \varepsilon_{k l}\right) .
$$

只有当 $E_{t e}^{*}=E_{t e}=0$ 时, 二者才相等. 由此可知

$$
\Delta Q_{b} \geqslant \Delta Q_{c} \text {. }
$$

加载路径 (d) 与 $(\mathrm{c})$ 的不同在于分叉时出现塑性膨胀, $E_{t m}^{*}$ 成为有限值. 一般来说, 当 $\left|E / E_{l m}^{*}\right| \gg 1$ 时,

$$
\frac{\nu}{1-2 \nu} \geqslant \frac{\nu-E / 3 E_{i m}^{*}}{1-2 \nu+E / E_{: m}^{*}} \rightarrow-\frac{1}{3}
$$

其中等号仅在 $E_{i m}^{*} \rightarrow \infty$ 时才成立. 又因

$$
\delta_{i j} \delta_{k l} \delta\left(d \varepsilon_{i j}\right) \delta\left(d \varepsilon_{k l}\right)=\left[\delta_{i j} \delta\left(d \varepsilon_{i j}\right)\right]^{2}>0,
$$

那么, 在相同的 $E_{t c}^{*}$ 值下, 附加塑性体膨胀以后

由此导致

$$
L_{i ; k l}^{*(d)} \delta\left(d \varepsilon_{i j}\right) \delta\left(d \varepsilon_{k l}\right) \leqslant L_{i, k l}^{*(c)} \delta\left(d \varepsilon_{i j}\right) \delta\left(d \varepsilon_{k l}\right) \text {. }
$$

$$
\Delta Q_{c} \geqslant \Delta Q_{d} .
$$

这一结果是以 (33) 式的成立为前提. 不难看出, 除非 $E / E_{t m}^{*}$ 是个很小的负值(这一情况实际 上可以除外), 否则(33)式总是有效的.

基于以上的各项推论，(23)式中的比较关系得到证实. 以 $Q_{a}$ 和 $Q_{b}$ 的关系为例可见， 要寻求分叉问题中的最小临界值, 只要在符合 (b) 型加载路径的 $Q_{b}$ 范围内进行即可. 这是 因为不可能有哪类扰动 $\delta \boldsymbol{V}$ 形式可以使 $Q$ 。从稳定态的正值出发, 在更小的临界值下先达 到零而 $Q_{b}$ 仍处于大于零的状态, 这一情况将与(23)式相违背. 依此类推, 一旦材料情况允许 (c) 路径出现, 就应该舍去 $Q_{b}$ 而在 $Q_{c}$ 范围内寻求最小临界值. 在有 (d) 路径出现的条件下, $Q_{a}$ 又优先于 $Q_{c}$.

为处理结构的塑性屈曲所采用的“弹性比较固体”或“一致加载”的概念 ${ }^{[g]}$ 就是基 于 $Q_{a} \geqslant$ $Q_{b}$. 屈曲时, 结构元件会有较大转动变形, 但应变量仍很小, 不足以造成材料内部损伤. 从材 料角度看, 还不具备出现(c)或 (d)类加载路径的条件, 自然也就不需要考虑后两种情况.

现有的材料研究表明，(17)和(18)式所归结的软化条件在大应变情况下是会出现的. 为 此，几何扰动与材料突变会同时出现。这就构成材料分叉与结构屈曲在加载路径上的本质区 别. 虽然材料不稳定性 (material instability) 的说法已常见于文献,但尚未指明它与结构屈 曲及不稳定性在概念上所存在的原则性不同.

利用这一新概念, 不仅能够解释为什么剪切带的实验临界值 ${ }^{[2]}$ 远高于理论值 ${ }^{[4]}$, 还可给出 在带内随同材料分布的突变而相应出现的速率扰动分布 ${ }^{[14]}$ 和模拟曲线型剪切带 ${ }^{[15]}$. 曲线型 剪切带与直线型带的区别在于，材料分布不仅沿带的法线方向有急剧突变而且在切线方向材 料的切线模量值也会有连续性变化. 这些算例都表明,材料内局部地区会出现速率分叉,在材 料条件许可的情况下,是和材料行为突变,即加载路径的转折相关联的. 


\section{四、结论}

1. 本文导出了具有一般性的一种可膨胀塑性本构方程. 它可以不限定于循环应力/应变 所耗散能量必须为正, 弹性与塑性无关, 加载面符合凸性等条件. 在无损伤 (小应变) 情况下, 它可以满足以上诸条件并简化为传统塑性力学中的 Prandt1-Reuss 关系. 进人有损伤（大应 变)阶段,结合微结构力学研究,材料实验和计算机模拟可以确定有关参数.

2. 利用本构方程具有一般性的特点, 可以讨论分叉点上材料的几种可能的加载路径. 结. 果证明, 在无损伤时该路径将遵守“一致加载”原则, 没有弹性卸载。一旦材料中允许出现更 “软”的突变时, 加载路径将顺应后一情况而转变. 此时, 速率分布的分叉是与材料行为的变化 相关联的.

\section{参考 文 献}

[ 1] Hancock, J. W. \& Cowling, M. J., Mez. Sci., 14(1980), 293.

[2] Anand, L., Spitzig. W. A., J. Mech. Phys. Solids, 28(1980), 113.

[3] Gurson, A. L., J. Eng. Mat. Technol, 99(1977), 2.

[4] Hill, R. \& Hutchinson, J. W., J. Mech. Phys. Solids, 23(1975). 239.

[5] Bushnell, D., Int. J., Solids Struczures, 10(1974), 1287.

[6] Li, G. C., Guennouni, T. \& Francois, D., Fatigue Fract. Engng. Mater. Struct., 12(1989), 105.

[7] Li, G. C., Acta Mechanica Solida Sinica (English edition), 2(1989), 175.

[8] Li, G. C. \& Howard, I. C., Advances in Fracture Research, Proc. Int. Conf. Fracture-6, 2(1984), 1191.

[ 9 ] Carroll, M. M., J. A. M. 54(1987), 15.

[10] Beremin, F. M., Met. Trans. A, 12A(1981), 723.

[11] Li, G. C. \& Howard, 1. C., J. Mech. Phys. Solids, 31(1983), 85.

[12] Xia, X. X., Yang, G. Y., Hong, Y. S. \& Li, G. C., Mechanical Behaviour of Materials-V, Proc. 5th Int. Conf. 1(1987), 199.

[13] Tvergaard, V., Int. J. Fracture, 17(1981), 389.

[14] Li, G. C., Acza Mechanica Sinica (English edition), 6(1990), 22.

[15] Li, G. C., Acta Mechanica Sinica (English edition),4(1988), 363. 